\title{
Common prefrontal activations during working memory, episodic memory, and semantic memory
}

\author{
Lars Nyberg $^{\mathrm{a}, *}$, Petter Marklund ${ }^{\mathrm{a}}$, Jonas Persson ${ }^{\mathrm{a}}$, Roberto Cabeza ${ }^{\mathrm{b}}$, \\ Christian Forkstam ${ }^{\mathrm{c}}$, Karl Magnus Petersson ${ }^{\mathrm{c}}$, Martin Ingvar ${ }^{\mathrm{c}}$ \\ a Department of Psychology, Umeå University, S-901 87 Umeå, Sweden \\ b Center for Cognitive Neuroscience, Duke University, Durham, USA \\ ${ }^{\mathrm{c}}$ Department of Clinical Neuroscience, Karolinska Institutet, Stockholm, Sweden
}

\begin{abstract}
Regions of the prefrontal cortex (PFC) are typically activated in many different cognitive functions. In most studies, the focus has been on the role of specific PFC regions in specific cognitive domains, but more recently similarities in PFC activations across cognitive domains have been stressed. Such similarities may suggest that a region mediates a common function across a variety of cognitive tasks. In this study, we compared the activation patterns associated with tests of working memory, semantic memory and episodic memory. The results converged on a general involvement of four regions across memory tests. These were located in left frontopolar cortex, left mid-ventrolateral PFC, left mid-dorsolateral PFC and dorsal anterior cingulate cortex. These findings provide evidence that some PFC regions are engaged during many different memory tests. The findings are discussed in relation to theories about the functional contribition of the PFC regions and the architecture of memory.
\end{abstract}

(C) 2002 Elsevier Science Ltd. All rights reserved.

Keywords: Prefrontal cortex; Memory; Positron emission tomography; Commonalities

\section{Introduction}

Functional brain imaging with positron emission tomography (PET) and functional magnetic resonance imaging (fMRI) have revealed a strong association between cognitive operations and activity in regions of the prefrontal cortex (PFC). For example, in a review of PET and fMRI studies [6], PFC regions were found to be part of the typical activation pattern for many different cognitive functions, including sustained attention, smell perception, written word recognition, verbal and spatial working memory, semantic memory, episodic memory, and conceptual priming.

In most prior studies, the focus has been on the role of specific PFC regions in specific cognitive domains. However, more recently it has been noted that regionally specific PFC activations show substantial similarities across cognitive domains $[8,26]$. In one recent analysis, Duncan and Owen [17] focused on five cognitive demands: response conflict, task novelty, number of elements in working memory, working-memory delay, and perceptual difficulty. They found that there was joint recruitment of three PFC

\footnotetext{
* Corresponding author. Tel.: +46-90-7866429; fax: +46-90-7866695

E-mail address: lars.nyberg@psy.umu.se (L. Nyberg).
}

regions for all five cognitive demands: mid-dorsolateral PFC, mid-ventrolateral PFC, and a dorsal part of the anterior cingulate cortex. These regions were seen as forming a common network recruited by as diverse challenges as response selection, working memory maintenance and stimulus recognition. It was furthermore noted that retrieval from episodic memory also tends to engage the same regions, and, in addition, that episodic retrieval showed a higher proportion of activations close to the frontal pole. These observations indicated that the common network is operating during episodic retrieval, but that additional processing associated with more anterior PFC regions also come into play.

The results from a recent fMRI study that directly contrasted working memory and episodic memory provide support for specific activation of frontopolar regions during episodic retrieval [7]. In contrast, another recent within-study fMRI comparison of working memory and episodic memory suggested that frontopolar activation was greater for working memory than for episodic memory [2], and frontopolar activation has also been associated with semantic monitoring [23]. Furthermore, both fMRI-studies [2,7] found evidence that dorsolateral PFC activation was stronger for working memory than for episodic memory. 
Thus, it is unclear whether dorsolateral PFC is engaged to a similar extent for different memory systems, and whether frontopolar activation is especially salient for episodic memory retrieval. At least in part, the unclear issues may have to do with different strategies for data analysis (betweenversus within-study comparisons) and with factors that are task specific.

The aim of the present study was to further explore similarities in regionally specific activations associated with different memory systems. This was accomplished by analysis of data from two PET experiments [27]. Across the two experiments, three tests were included for each of three memory systems: episodic memory, working memory, and semantic memory. This design allowed analysis of regional activations that are common for a wide range of memory tasks.

\section{Methods}

\subsection{Experimental tasks}

The tasks that were used for the three systems are summarized in Table 1. Working memory was measured with 1and 2-back tasks [3] and with a random-number generation task (cf. [32]). In the 1- and 2-back tasks, subjects were instructed that a sequence of words was to be presented and that their task was to decide for each word whether it was the same as the one presented one or two items earlier in the list. In the random-number generation task, subjects were instructed that each time a "?" appeared on the screen their task was to randomly generate a number between 1 and 10 . They were told not to mention the same number twice in succession and to use all numbers between 1 and 10 before starting over again.

Episodic memory was measured with yes/no recognition (e.g. [28]), category-instance cued recall [36], and autobiographical memory [11]. In the recognition test, subjects were shown a mixed list of nine non-studied words and nine words from an intentional encoding session and were asked to say "yes" when they recognized a word and "no" when they thought a non-studied word was presented. In the cued-recall test, subjects were presented cue words (e.g. AUTHOR) and were asked to recall targets (e.g. STRINDBERG) from a previous study session (or say "no" if they could not recall the target). In the autobiographical test, subjects were presented cue words (e.g. VACATION) and were asked to use these for remembering personal events that could be related to each cue. They responded by saying one word that described their memory (e.g. "GREECE") or "no" if they could not come up with a personal memory.

Semantic memory was measured with living/non-living classification [19] fact retrieval (cf. [36]), and synonym generation (cf. [20]). In the living/non-living task, subjects were presented a list of words and decided if the words referred to living or non-living things. In fact retrieval, subjects were shown a series of cue words (e.g. AUTHOR) and were asked to retrieve factual information associated with each cue. They responded by saying one word that related to the factual information (or said "no"). In synonym generation, subjects were presented a series of words. For each word they were instructed to generate a different word with similar meaning (e.g. VACATION-HOLIDAY) or with a strong semantic association to the cue word (e.g. CARVOLVO).

The experiments also included a baseline reading condition. Subjects were told that a series of words was going to be presented and that their task was to read each word aloud. They were explicitly told that these words were not part of any test and that they did not have to memorize them.

\subsection{Experimental procedure}

The procedure for stimulus presentation and responding was the same for all tasks, and involved presentation of single items on a computer screen placed above the subjects' heads and responding by saying one word per stimulus (stimuli were words in all conditions except for random-number generation in which a series of "?" was presented). The presentation rate was $3 \mathrm{~s}$ (inter-stimulus interval $=2 \mathrm{~s}$ ). Each experimental condition included 18 stimuli, 12 of which were presented during the scan interval.

Both experiments included seven experimental conditions, each presented and scanned twice (i.e. 14 scans/ experiment). Experiment 1 included the following tasks: 2-back, random-number generation, recognition, cued recall, living/non-living, fact retrieval, and the baseline reading condition. Experiment 2 included: 1-back, cued recall, autobiographical memory, fact retrieval, synonym generation, and the reading baseline (a second baseline condition was also included but will not be considered here). The experimental tasks were presented in a counterbalanced order across subjects with the restriction that all conditions were performed before the replications were presented. An experimenter recorded the verbal responses (accuracy was $>90 \%$ in all tasks).

Table 1

Experimental tasks

\begin{tabular}{lll}
\hline System & Task & \\
\hline Working memory & 1-Back & 2-Back \\
Episodic memory & Yes/no recognition & Category cued recall \\
Semantic memory & Living/non-living & Fact retrieval \\
\hline
\end{tabular}


(a)

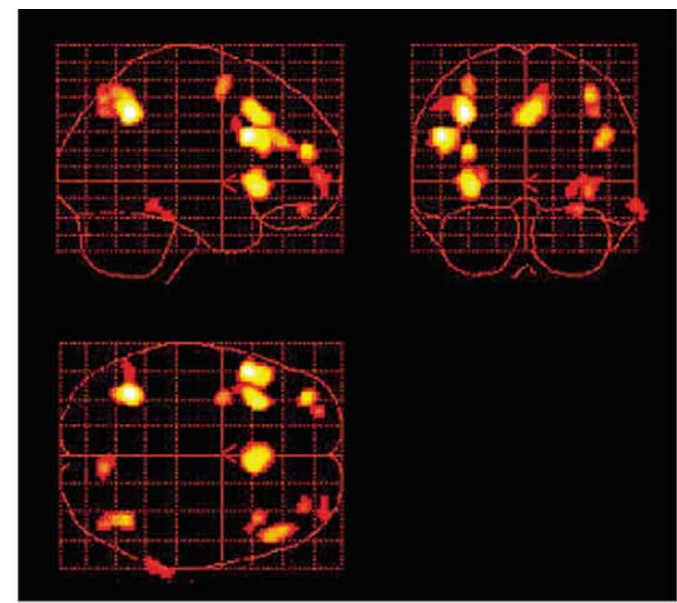

(b)

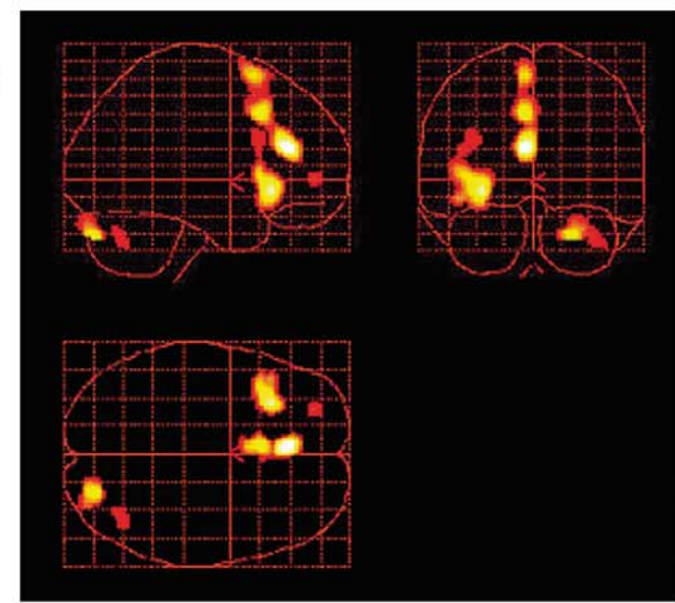

(c)

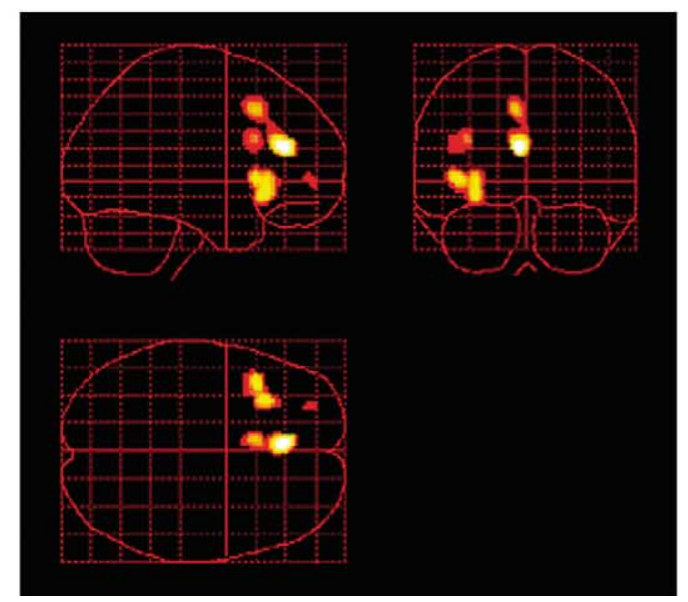

(d)

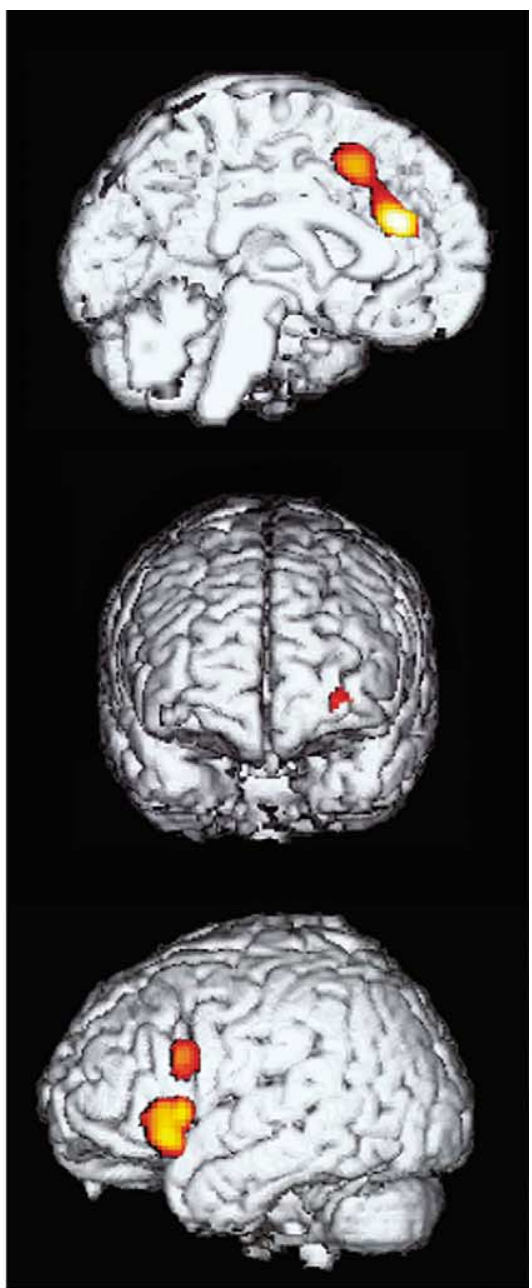

Fig. 1. (a) Increased activity in Experiment 1 during memory compared to baseline. Significant activations were observed in left inferior parietal cortex $(x, y, z=-36,-56,42$, Brodmann area (BA) 40), mid-dorsolateral PFC $(x, y, z=-50,16,28$, BA 9/46), mid-ventrolateral PFC ( $x, y, z=-32,20$, -2 , BA 45/47), dorsal anterior cingulate cortex $(x, y, z=2,20,40$, BA 24/32), left middle frontal gyrus ( $x, y, z=-34$, 50, 20, BA 10), right superior parietal cortex $(x, y, z=38,-58,52$, BA 7), right middle frontal gyrus $(x, y, z=48,30,28$, BA 9/46), left middle frontal gyrus $(x, y, z=-34,0$, 58, BA 6), precuneus $(x, y, z=8,-72,50$, BA 7), right inferior frontal gyrus $(x, y, z=38,20,-4$, BA 47), right middle/superior frontal gyrus ( $x$, $y, z=32,48,-16$, BA 11), left frontopolar cortex $(x, y, z=-26,58,2$, BA 10), right inferior middle temporal gyrus (66, -42, -14 , BA 20/21), and right superior frontal gyrus $(28,60,-4$, BA 10). Activations thresholded at $P<0.05$ corrected, extent threshold $>25$ voxels; (b) Increased activity in Experiment 2 during memory compared to baseline. Significant activations were found in dorsal anterior cingulate cortex $(x, y, z=-4,18,40$, BA 24/32), left mid-ventrolateral PFC ( $x, y, z=-30,24,-6$, BA 47), left frontopolar cortex $(x, y, z=-26,50,2$, BA 10$)$, and right cerebellum ( $x, y$, $z=22,-82,-34)$. Activations thresholded at $P<0.05$ corrected, extent threshold $>25$ voxels; (c) Overlapping activations common to both experiments were found in two distinct but contiguous areas of the dorsal anterior cingulate cortex $(x, y, z=-4,34,20, \mathrm{BA} 24 / 32, Z=6.93$, and $-6,18,44, \mathrm{BA}$ $24 / 32, Z=5.72)$, mid-ventrolateral PFC $(-30,24,-8$, BA $47, Z=6.11)$, mid-dorsolateral PFC $(-36$, 18, 26, BA 46/9, $Z=5.42)$, and frontopolar cortex $(-26,50,2$, BA 10, $Z=4.91)$. Activations thresholded at $P<0.05$ corrected, extent threshold $>10$ voxels; (d) Approximate localization of the four common prefrontal regions: top, dorsal part of the anterior cingulate cortex; middle, left frontopolar cortex; bottom, left mid-ventrolateral PFC and left mid-dorsolateral PFC. Activations were rendered on a brain volume transformed into standardized space. Note that the anatomical localizations are approximations for illustrative purposes. 
Table 2

Common prefrontal activations across memory tests

\begin{tabular}{|c|c|c|c|c|c|}
\hline \multirow[t]{3}{*}{ Test } & \multicolumn{5}{|c|}{ Prefrontal activation peaks ${ }^{\mathrm{a}}$} \\
\hline & \multicolumn{2}{|c|}{ Anterior cingulate } & \multirow{2}{*}{$\begin{array}{l}\text { Mid-dorsolateral } \\
(-36,18,26)\end{array}$} & \multirow{2}{*}{$\begin{array}{l}\text { Frontopolar } \\
(-26,50,2)\end{array}$} & \multirow{2}{*}{$\begin{array}{l}\text { Mid-ventrolateral } \\
(-30,24,-8)\end{array}$} \\
\hline & $(-4,34,20)$ & $(-6,18,44)$ & & & \\
\hline \multicolumn{6}{|l|}{ Working memory } \\
\hline 1-Back & $(-6,36,20) z=3.69$ & $(-6,12,44) z=2.63$ & $(-36,16,28) z=2.34$ & $(-28,54,-4) z=3.51$ & $(-42,18,4) z=2.58$ \\
\hline 2-Back & $(-6,30,22) z=2.04$ & $(-4,16,42) z=4.93$ & $(-44,20,26) z=4.33$ & $(-28,52,2) z=2.67$ & $(-32,24,6) z=4.13$ \\
\hline Random number-generation & $(-6,30,24) z=3.82$ & $(-2,18,40) z=5.78$ & $(-46,22,24) z=4.67$ & $(-28,52,2) z=3.29$ & $(-38,16,2) z=4.67$ \\
\hline \multicolumn{6}{|l|}{ Episodic memory } \\
\hline Recognition $^{\mathrm{b}}$ & $(-8,42,22) z=1.45$ & $(-2,22,38) z=3.72$ & $(-46,22,24) z=2.96$ & $(-28,52,2) z=2.17$ & $(-38,16,2) z=4.40$ \\
\hline Cued recall (Experiment 1) & $(-4,34,20) z=3.51$ & $(-4,18,40) z=4.07$ & $(-46,14,26) z=3.19$ & $(-28,52,2) z=2.37$ & $(-34,18,-6) z=5.11$ \\
\hline Cued recall (Experiment 2) & $(-6,34,22) z=6.57$ & $(-4,20,38) z=3.81$ & $(-36,20,24) z=5.43$ & $(-26,54,0) z=4.50$ & $(-30,22,-8) z=6.51$ \\
\hline Autobiographical & $(-2,30,20) z=5.74$ & $(-6,18,42) z=6.42$ & $(-44,18,18) z=5.44$ & $(-28,50,0) z=5.03$ & $(-42,18,0) z=6.90$ \\
\hline \multicolumn{6}{|l|}{ Semantic memory } \\
\hline Fact retrieval (Experiment 1) & $(-8,34,22) z=3.86$ & $(-4,22,36) z=5.09$ & $(-40,20,22) z=5.00$ & $(-26,54,0) z=1.72$ & $(-32,24,-6) z=5.32$ \\
\hline Fact retrieval (Experiment 2) & $(-4,36,20) z=5.44$ & $(-6,22,42) z=5.47$ & $(-46,16,22) z=5.09$ & $(-26,48,2) z=3.67$ & $(-30,26,-8) z=5.89$ \\
\hline Synonym generation & $(-4,36,18) z=6.13$ & $(-6,18,46) z=5.58$ & $(-46,14,20) z=4.84$ & $(-28,52,0) z=4.23$ & $(-30,24,0) z=5.47$ \\
\hline Living/non-living & $(-4,32,28) z=2.38$ & $(-4,24,38) z=2.23$ & $(-46,14,26) z=2.57$ & $(-28,54,-4) z=0.58$ & $(-34,22,-10) z=3.79$ \\
\hline
\end{tabular}

${ }^{\text {a } C o o r d i n a t e s ~}(x, y, z)$ according to Talairach and Tournoux, negative left/posterior/inferior.

${ }^{\mathrm{b}}$ Significant for one of the two anterior cingulate cortex peaks. 


\subsection{Participants}

The two experiments included a total of 29 right-handed healthy male subjects (Experiment 1: $N=15$, age $=28 \pm 7$ years; Experiment 2: $N=14$, age $=24 \pm 4$ years). The subjects were prescreened and none used any medication, had a history of drug abuse (including nicotine), head trauma, neurological or psychiatric illness, or a family history of neurological or psychiatric illness. All had university level education. The local Ethics and Radiation Safety committees at the Karolinska Hospital approved the study, and all the subjects gave written informed consent.

\subsection{Data acquisition}

Regional cerebral blood flow (rCBF) was measured with a 3D ECAT EXACT HR PET scanner and bolus injections of $\mathrm{H}_{2}{ }^{15} \mathrm{O}$. Each subject underwent $14 \mathrm{rCBF}$ measurements. The PET scanner was used in 3D-sampling mode producing $60 \mathrm{~s}$ tracer uptake images. The different tasks were started at the time of tracer injection and the scanning was automatically initiated when the activity level in the brain exceeded a predetermined level above background. Scatter correction was done and a 2D-transmission scan was used for attenuation correction.

\subsection{Statistical analysis}

SPM99 (http://www.fil.ion.ucl.ac.uk/) was used for image preprocessing and analysis. The PET-images were realigned, spatially normalized and transformed into a common approximate Talairach stereotactic space as defined by the SPM99 PET template, 3D-Gaussian filtered $(10 \mathrm{~mm}$ FWHM), and proportionally scaled to account for global confounders.

In order to test specific null-hypotheses, linear contrasts of different conditions were created, generating t-fields (SPM [t]). First, commonalities in activations across memory conditions were identified by a weighted contrast of all memory tasks relative to baseline for each of the two experiments. Next, regions that showed significantly increased activity during memory compared to baseline in both experiments were identified by inclusive masking, where the activation map for memory versus reading in Experiment 1 served as a mask for the corresponding activation map in Experiment 2 (thresholded at $P<0.05$, corrected). $P$-values relating to significant activations were corrected for multiple non-independent comparisons based on the theory of smooth t-fields [43].

In a second step, we examined whether commonly activated regions from the masked analysis were significantly activated for each individual memory task, or whether the overall effect was driven by a subset of tasks. This was done by using the common regions from the masked analysis as volumes of-interest (VOI's) in pairwise comparisons of each memory test with the baseline.

\section{Results}

The overall memory-baseline contrast in each experiment revealed several significant increases in regional activity $(P<0.05$ corrected; Fig. 1a and b). Comparison of the two activation maps by the masking procedure showed that four regional effects were common to both contrasts. These regions were located in dorsal anterior cingulate cortex, left frontopolar cortex, left mid-dorsolateral PFC and left mid-ventrolateral PFC (Fig. 1c and d). The activation in the anterior cingulate cortex consisted of two peaks, both of which fell in the dorsal cognitive subdivision [5].

In the second step, where each memory task was contrasted with the reading baseline condition, the results showed that, with one exception, the four common regions were consistently activated $(P<0.05)$ (Table 2$)$. The exception was that the frontopolar activation was non-significant for the living/non-living classification task. Given that the frontopolar VOI was quite small, we conducted a whole-brain contrast of the living/non-living task with the baseline condition. The corresponding activation map included an activation $6 \mathrm{~mm}$ anterior of the frontopolar VOI $(x, y, z=-26,58,2 ; Z=2.31)$. Thus, although outside the VOI, frontopolar activation was seen also for the living/non-living task.

\section{Discussion}

There is increasing evidence for commonalities in patterns of PFC activity associated with different cognitive tasks $[6,10,14,16-18,26,33]$. The present analyses of regional activity associated with tests of working memory, episodic memory, and semantic memory provide additional evidence by converging on a general involvement of four PFC regions across memory tasks. These were located in left frontopolar cortex, left mid-ventrolateral PFC, left mid-dorsolateral $\mathrm{PFC}$ and dorsal anterior cingulate cortex.

There are several possible explanations for a consistent memory-related increase in activity in these regions. One obvious factor is that, relative to the reading-baseline task, all of the memory tasks should have involved increased demands on executive processing or cognitive control [38]. Indeed, for all observed regions, functional accounts have been proposed that relate to executive processing or cognitive control.

Increased memory-related activity in mid-ventrolateral PFC has been related to updating and maintaining the contents of working memory [18,24,31]. By this view, working-memory processes contribute also to long-term memory tasks (see also [41]). A related functional account holds that mid-ventrolateral PFC has a general role in memory by mediating active encoding and retrieval of information [29]. Mid-dorsolateral PFC has been suggested to play a crucial role in cognitive operations that require active selection, monitoring and acting upon material held 
within working memory $[13,15,18]$. Moreover, dorsolateral PFC has also been implicated as being critical for the evaluation of externally attended-to information [10], which all memory tasks in the present study likely demanded. A general memory-related activity increase in polar PFC has been related to higher-order control of goals, and processes employed to achieve the goals [18] (see also [21]). The observed pattern of results would also be consistent with a role of polar PFC in evaluation of self-generated information [10]. Finally, increased activity in the anterior cingulate cortex has been related to cognitive control and effortful task completion $[9,12,22]$.

Although focus in this study was on similarities in brain activity across memory tasks, it should be emphasized that there are many observations of differential regional activity depending on type of memory task [27]. For example, consistent with a systems-perspective on memory function, specific activation patterns within PFC have been revealed for working memory, episodic memory and semantic memory $[2,36]$. Differential brain activity has also been observed with regard to the nature of the component processes engaged by different memory tasks $[4,28,29,42]$. In addition, degree of activity within specific PFC regions has been found to be affected by factors such as task difficulty, task novelty and errors in task performance [12]. For example, activity in the anterior cingulate cortex has been found to be positively correlated with degree of task difficulty [1].

In conclusion, the present results suggest that certain processes are common to several different tests of working memory, episodic memory and semantic memory. This is well in line with the view that human memory is composed of multiple processing components, some common to many tests and others specific to certain tests $[9,25,34]$. The results may appear more difficult to reconcile with a multiple-memory systems account (e.g. [37,40]). However, it has been stressed that systems operate in terms of processes, some that are common across systems and some that are unique for specific systems [35,39]. As such, the components-of-processing account and the multiple memory systems account appear quite similar (cf. [30]). Importantly, though, up to recently, little has been known about processes that are shared by many memory tests. The present and related findings provide some initial clues as to the nature of such common processes.

\section{Acknowledgements}

This work was supported by a grant from the Swedish Research Council to LN. MI, KMP, \& CF were supported by grants from the Swedish Research Council (8276), the Karolinska Institute, the Knut and Alice Wallenberg foundation, and the Petrus and Augusta Hedlund Foundation. The authors gratefully acknowledge the staff at the PET facility.

\section{References}

[1] Barch DM, Braver TS, Nystrom LE, Forman SD, Noll DC, Cohen JD. Dissociating working memory from task difficulty in human prefrontal cortex. Neuropsychologia 1997;35(10):1373-80.

[2] Braver TS, Barch DM, Kelley WM, Buckner RL, Cohen NJ, Miezin FM, et al. Direct comparison of prefrontal cortex regions engaged by working and long-term memory tasks. Neuroimage 2001;14:48-59.

[3] Braver TS, Cohen JD, Nystrom LE, Jonides J, Smith EE, Noll DC. A parametric study of prefrontal cortex involvement in human working memory. Neuroimage 1997;5:49-62.

[4] Buckner RL, Wheeler ME. The cognitive neuroscience of remembering. Nature Neuroscience Reviews 2001;2:624-34.

[5] Bush G, Luu P, Posner MI. Cognitive and emotional influences in anterior cingulate cortex. Trends in Cognitive Sciences 2000;4(6):215-22.

[6] Cabeza R, Nyberg L. Imaging cognition. Part II. Empirical review of 275 PET and fMRI studies. Journal of Cognitive Neuroscience 2000;12(1): $1-47$.

[7] Cabeza R, Dolcos F, Graham R, Nyberg L. Similarities and differences in the neural correlates of episodic memory retrieval and working memory. Neuroimage 2001;16:317-30.

[8] Cabeza R, Nyberg L. Seeing the forest through the trees: the cross-function approach to imaging cognition. In: Zani A, Proverbio A, editors. The cognitive electrophysiology of mind and brain. San Diego: Academic Press; in press.

[9] Carter CS, Braver TS, Barch DM, Botvinick MM, Noll DC, Cohen JD. Anterior cingulate cortex, error detection, and the online monitoring of performance. Science 1998;280:747-9.

[10] Christoff K, Gabrieli JDE. The frontopolar cortex and human cognition: evidence for a rostrocaudal hierarchical organization within the human prefrontal cortex. Psychobiology 2000;28:168-86.

[11] Conway MA, Turk DJ, Miller SL, Logan J, Nebes RD, Meltzer CC, et al. A positron emission tomography (PET) study of autobiographical memory retrieval. Memory 1999;7:679-702.

[12] Dehaene S, Kerszberg M, Changeux JP. A neuronal model of a global workspace in effortful cognitive tasks. Proceedings of the National Academy of Science in the USA 1998;24:14529-34.

[13] D'Esposito M. Functional neuroimaging of working memory. In: Cabeza R, Kingstone A, editors. Handbook of functional neuroimaging of cognition. Cambridge, MA: MIT Press; 2001, p. 293-327.

[14] D'Esposito M, Ballard D, Aguirre GK, Zarahn E. Human prefrontal cortex is not specific for working memory: a functional MRI study. Neuroimage 1998;8:274-82.

[15] D'Esposito M, Aguirre GK, Zarahn E, Ballard D, Shin RK, Lease $\mathrm{J}$, et al. Functional MRI studies of spatial and non-spatial working memory. Cognitive Brain Research 1998;7:1-13.

[16] Duncan J. An adaptive coding model of neural function in prefrontal cortex. Nature Neuroscience Reviews 2001;2:820-9.

[17] Duncan J, Owen AM. Common regions of the human frontal lobe recruited by diverse cognitive demands. Trends in Neuroscience 2000;23:475-83.

[18] Fletcher PC, Henson RNA. Frontal lobes and human memory: insights from functional neuroimaging. Brain 2001;124(124):849-81.

[19] Kapur S, Craik F, Tulving E, Wilson A, Houle S, Brown G. Neuroanatomical correlates of encoding in episodic memory: levels of processing effect. Proceedings of the National Academy of Science in the USA 1994;91(6):2008-11.

[20] Klein D, Milner B, Zatorre RJ, Meyer E, Evans AC. The neural substrates underlying word generation: a bilingual functional-imaging study. Proceedings of the National Academy of Science in the USA 1995;92:2899-903.

[21] Koechlin E, Corrado G, Pietrini P, Grafman J. Dissociating the role of the medial and lateral anterior prefrontal cortex in human planning. Proceedings of the National Academy of Science in the USA 2000;97(13):7651-6. 
[22] MacDonald AW, Cohen JD, Stenger VA, Carter CS. Dissociating the role of the dorsolateral prefrontal and anterior cingulate cortex in cognitive control. Science 2000;288:1835-8.

[23] MacLeod AK, Buckner RL, Miezin FM, Petersen SE. Right anterior prefrontal cortex activation during semantic monitoring and working memory. Neuroimage 1998;7(1):41-8.

[24] Milner B, Petrides M, Smith ML. Frontal lobes and the temporal organization of memory. Human Neurobiology 1985;4:137-42.

[25] Moscovitch M. Memory and working-with-memory: a component process model based on modules and central systems. Journal of Cognitive Neuroscience 1992;4:257-67.

[26] Nyberg L, Cabeza R. Similarities in brain activity during working memory, semantic memory, and episodic memory: implications for theories of memory. In: Algarabel A, Pitarque T, Bajo S, Gathercole S, Conway M, editors. Theories of memory. London: Psychology Press; in press.

[27] Nyberg L, Forkstam C, Petersson K, Cabeza R, Ingvar M. Brain imaging of human memory systems: between-systems similarities and within-systems differences. Cognitive Brain Research 2002;13: 281-92.

[28] Nyberg L, Tulving E, Habib R, Nilsson L-G, Kapur S, Houle S, et al. Functional brain maps of retrieval mode and recovery of episodic information. Neuroreport 1995;7:249-52.

[29] Owen A, Lee A, Williams E. Dissociating aspects of verbal working memory within the human frontal lobe: further evidence for a "process-specific" model of lateral frontal organization. Psychobiology 2000;28:146-55.

[30] Parkin AJ. Component processes versus systems: is there really an important difference? In: Foster JK, Jelicic M., editors. Memory: systems, process, or function? New York: Oxford University Press; 1999, p. 273-87.

[31] Petrides M. Frontal lobes and behavior. Current opinion in neurobiology 1994;4:207-11.

[32] Petrides M, Alivisatos B, Meyer E, Evans AC. Functional activation of the human frontal-cortex during the performance of verbal working memory tasks. Proceedings of the National Academy of Science in the USA $1993 ; 90: 878-82$.

[33] Ranganath C, Johnson M, D'Esposito M. Prefrontal activity associated with working memory and episodic long-term memory. Neuropsychologia, this issue.

[34] Roediger HL, Buckner RL, McDermott KB. Components of processing. In: Foster JK, Jelicic M, editors. Memory: systems, process, or function? New York: Oxford University Press; 1999, p. 31-65.

[35] Schacter DL, Wagner AD, Buckner RL. Memory systems of 1999. In: Tulving E, Craik FIM, editors. The oxford handbook of memory. New York: Oxford University Press; 2000, p. 627-43.

[36] Shallice T, Fletcher P, Frith CD, Grasby P, Frackowiak RSJ, Dolan RJ. Brain regions associated with acquisition and retrieval of verbal episodic memory. Nature 1994;368:633-5.

[37] Squire L. Declarative and non-declarative memory-multiple brain systems supporting learning and memory. Journal of Cognitive Neuroscience 1992;4(3):232-43.

[38] Sylvester C, Wager T, Lacey S, Jonides J, Smith E, Hernandez L, Nichols T. Switching attention and resolving interference: fMRI measures of executive functions. Neuropsychologia, this issue.

[39] Tulving E. Study of memory: processes and systems. In: Foster JK, Jelicic M, editors. Memory: systems, process, or function? New York: Oxford University Press; 1999, p. 11-30.

[40] Tulving E, Schacter DL. Priming and human-memory systems. Science 1990;247:301-6.

[41] Wagner AD. Working memory contributions to human learning and remembering. Neuron 1999;22:19-22.

[42] Wagner AD. Cognitive control and episodic memory: contributions from prefrontal cortex. In: Squire LR, Schacter DL, editors. Neuropsychology of memory, 3rd ed. London: Psychology Press; 2002, p. 174-92.

[43] Worsley KJ, Marrett S, Neelin P, Vandal AC, Friston KJ, Evans AC. A unified statistical approach for determining significant signals in images of cerebral activation. Human Brain Mapping 1996;1(4): $58-73$. 